\title{
The Origin of Cellular Life and
}

\section{Biosemiotics}

\section{Attila Grandpierre}

\section{Biosemiotics}

ISSN 1875-1342

Biosemiotics

DOI 10.1007/s12304-013-9173-9

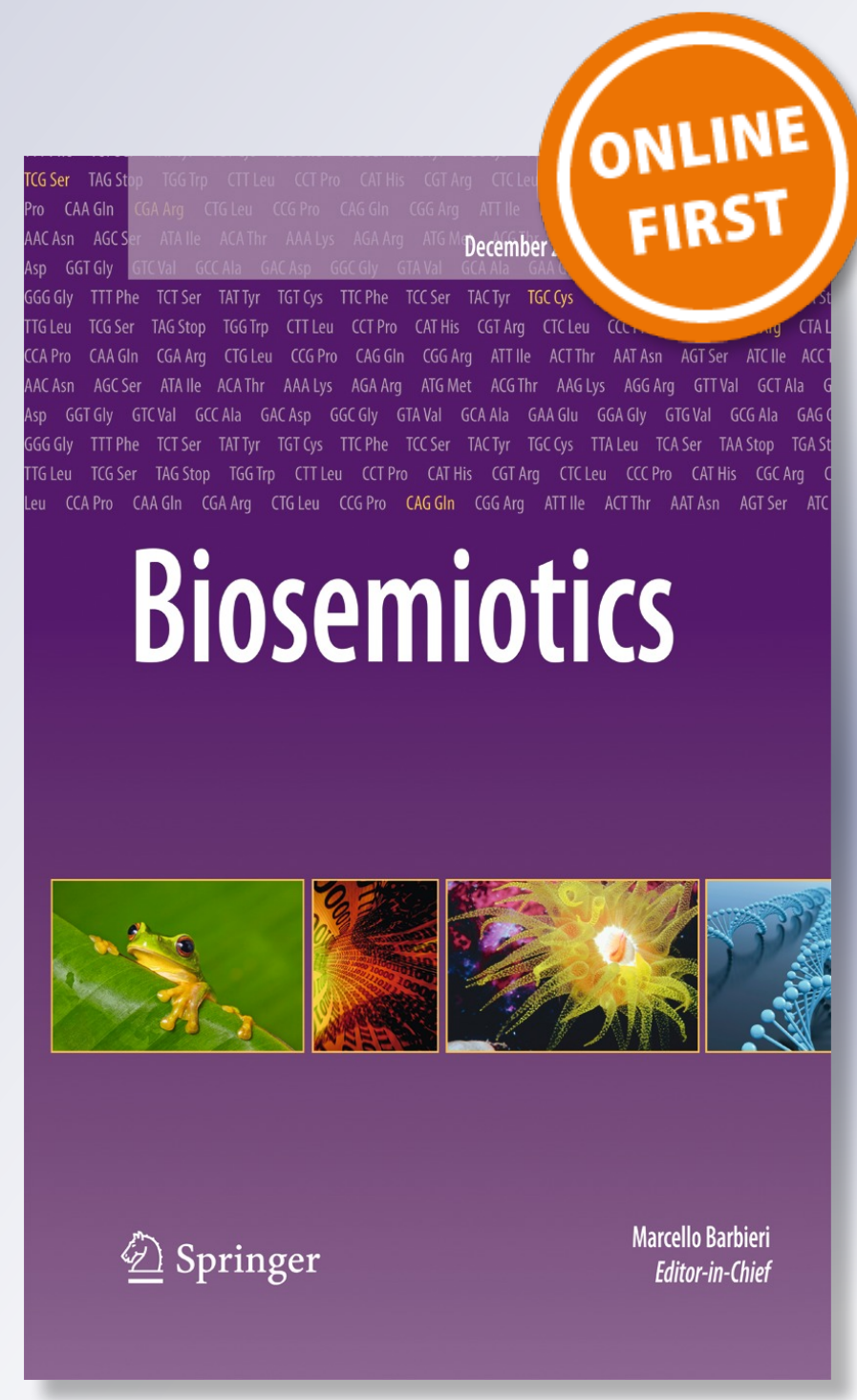

照 Springer 
Published in: Biosemiotics, Published online: 24 February 2013

http://link.springer.com/article/10.1007\%2Fs12304-013-9173-9

DOI 10.1007/s12304-013-9173-9

ORIGINAL PAPER

\title{
The Origin of Cellular Life and Biosemiotics ${ }^{1}$ Attila Grandpierre
}

Konkoly Observatory of the Hungarian Academy of Sciences, H-1525 Budapest, P. O. Box 67, Hungary

e-mail: grandp@iif.hu

Received: 21 September 2012 / Accepted: 13 December 2012 \# Springer Science+Business Media Dordrecht 2013

\begin{abstract}
Recent successes of systems biology clarified that biological functionality is multilevel. We point out that this fact makes it necessary to revise popular views about macromolecular functions and distinguish between local, physico-chemical and global, biological functions. Our analysis shows that physico-chemical functions are merely tools of biological functionality. This result sheds new light on the origin of cellular life, indicating that in evolutionary history, assignment of biological functions to cellular ingredients plays a crucial role. In this wider picture, even if aggregation of chance mutations of replicator molecules and spontaneously self-assembled proteins led to the formation of a system identical with a living cell in all physical respects but devoid of biological functions, it would remain an inanimate physical system, a pseudo-cell or a zombie-cell but not a viable cell. In the origin of life scenarios, a fundamental circularity arises, since if cells are the minimal units of life, it is apparent that assignments of cellular functions require the presence of cells and vice versa. Resolution of this dilemma requires distinguishing between physico-chemical and biological symbols as well as between physico-chemical and biological information. Our analysis of the concepts of symbol, rule and code suggests that they all rely implicitly on biological laws or principles. We show that the problem is how to establish physico-chemically arbitrary rules assigning biological functions without the presence of living organisms. We propose a solution to that problem with the help of a generalized action principle and biological harnessing of quantum uncertainties. By our proposal, biology is an autonomous science having its own fundamental principle. The biological principle ought not to be regarded as an emergent phenom- enon. It can guide chemical evolution towards the biological one, progressively assigning greater complexity and functionality to macromolecules and systems of macromolecules at all levels of organization. This solution explains some perplexing facts and posits a new context for thinking about the problems of the origin of life and mind.
\end{abstract}

Keywords molecular functions - cellular functions - systems biology — physical symbols biological symbols - quantum vacuum - emergence - supervenience - mind

\footnotetext{
${ }^{1}$ Special Issue "Origins of Mind" edited by Liz Stillwaggon Swan and Andrew M. Winters

A. Grandpierre (凶)

Konkoly Observatory of the Hungarian Academy of Sciences, 1525 Budapest, P. O. Box 67, Hungary e-mail: grandp@iif.hu
} 


\section{Introduction}

In the last decades genetic essentialism, the idea of a determinate genetic program in the DNA controlling the development and functioning of the organism, rather like the digital code of a computer program, was seductive (Bouchard 2004; Noble 2010; Dar-Nimrod and Heine 2011; Rosoff 2012). Nevertheless, recently it was shown that biological functionality is multilevel (Noble 2008a). Claude Bernard's concept of the constancy of the internal environment implies the existence of control processes to achieve this. It has been well known for a long time that "developmental controls in cells may be executed by "ordinary" molecules to which we give titles, such as activator, repressor, or hormone, but the control value of these molecules is not an inherent chemical property; it's a complex relation established by a collective hierar- chical organization, requiring the whole organism" (Pattee 1973). Noble has pointed out that the control of the 'milieu interieur' meant not that the individual molecules did anything measurably different from what they would do in non-living systems, but rather that the ensemble behaves in a controlled way, the controls being those that maintain the constancy of the internal environment. Such functionality is attributable to the organism as a whole and it controls all the other levels. For, in truth, the stretches of DNA that we now call genes do nothing on their own. They are simply databases used by the organism as a whole (Noble 2008a; see also Kawade 1992; Hoffmeyer 1998). Keeping in mind that finding, our point here is that in the process of cellular biogenesis the production of DNA, RNA and proteins-even if these were successfully made by abiotic processes, and their assembly suitable — is not enough to produce a viable cell, because the most vital ingredient is missing: suitable multilevel control securing biological organization, coordinating biological activities. Accepting that DNA is simply a database used by the organism as a whole shifts the basic level of determinations from the DNA to the cell as a whole. Our point here is that in the context of cellular biogenesis a viable cell cannot arise from even a well-prepared database and all the other tools. If biological functions are multilevel, and determined from the cellular level, then cells cannot arise on physico-chemical grounds since the cells are needed to determine the biological functions necessary for their viability. This is our first argument against a merely physico-chemical origin of cellular life (we call it the multilevel argument).

It is a general view that the three-dimensional structure of a protein defines not only its size and shape, but also its function (Tachibana et al. 2010). Yet we point out that so-called higher-level functions, or, as we propose to call them here, biological functions, are to be distinguished from lower-level functions that we propose to call elementary, local and physico-chemical functions. In order to clarify this issue, let us consider what is meant by the term biological function. As Morton Beckner (1969) defined it: "Functional ascriptions describe the role played by a part or process in the activities of a larger or more inclusive system. Standard examples are the ascriptions of roles to the organs, tissues, cellular parts, biochemical processes, and so on, in the growth, regulation, maintenance, and reproduction of organisms. In general, function is always function in a whole system." Man-made machines also have functions; these are pre-fixed, mechanical ones. Thus we suggest it is useful to distinguish between machine-like mechanical functions and genuine biological functions. Biological functions are always flexible, manifesting equifinality, since organisms commonly have alternative means of performing the same function (Beckner 1969, 155). Moreover, not all biological functions are pre-fixed like the functions of organs, since not only on the long timescale of evolution, but also on the shorter timescale of the individual organism or a cell, novel biological functions are regularly acquired (Shapiro 2011, 1-5, 56). The functions of the living organism typically depend upon the coherent operations of molecules by the millions, of hundreds or even thousands of different kinds, and marshaled into functional organization by a hierarchy of controls (Harold 2001, 4) that extends over distances orders of magnitude larger (ibid., 66) than the individual molecules themselves. As Noble (2008b) noted, high- level functions depend on DNA and the rest of the cell. As Hoffmeyer formulated it, "the most primitive concept of function implies improving fitness of an organism" (Hoffmeyer 2001). Our point is that, as the above arguments indicate, biological functions are always determined at the organismal, global, integral level, while, in contrast, physico-chemical functions are always determined locally, given by the structure of the molecules.

We consider here the origin of the first cell. It will be useful to take an example. An elementary, physico-chemical function of some biomolecules is bondmaking (Barbieri 2008). Certainly, bondmakers have physico-chemical properties (like chemical composition, sequence, or spatial shape) suitable for bondmaking. Considering the origin of the first knife, a sharp chopper that has the form, chemical composition and size suitable to function as a knife, can cut. Just as a knife has a function, a bondmaker or a washing machine has a similar type of function, namely, determined by its structure. This is what we call a physico-chemical function, recognizing that this function is completely 
determined by local, structural physico-chemical proper- ties. Nevertheless, in a living cell bondmaking serves the permanently self-renewing system of cellular functions. Similarly, although the physical function of the chopper is completely determined by the physico-chemical properties of the chopper, it became a knife only when a Homo erectus had the aim to cut and, searching for a suitable tool, realized the potential suitability of the chopper to cut. In a strict sense, the chopper, even in the presence of all its suitable physical properties to cut, like sharpness and hardness, did not have function at all until it was coupled to a purpose. The origin of the first knife therefore occurs not at the natural abiogenetic production of the first sharp object, but in the period when the early Homo erectus discovered that sharp objects are suitable to cut and started to use them as tools to cut. We suggest that the actual realization of bondmaking, its timing as well as its proper, time-variable biological context that couples bondmaking to the coherent operations of millions of molecules serving cellular function must be suitably determined by the cell. As Harold formulated the relation between local and global determinations: Briefly, the genes specify What; the cell as a whole directs Where and When; and at the end of the day, it is the cell that usually supplies the best answer to the question Why (Harold 2001, 82). Every act of bondmaking occurs in a biological context, first of all, in functional relations with the hierarchy of biological functions or aims. This means that bondmaking will occur in a living cell only with the assistance of biological organization, a system of biological couplings that determines the timing and synchrony, the initiation and coordination of cellular processes.

If so, then the local, physico-chemical function is only a part of the global biological function. The bondmaker is merely a tool in the process of biological organization that extends to the whole of the cell, similarly to a knife that is merely a tool in the hand of its user. Therefore, in reality the biological function is the precondition of the realization of physical functions. This means that the origin of cellular life begins with the origin of its biological functions. Yet, while the origin of physico-chemical functions is a problem that at least conceptually may seem to be clear, the origin of biological functions seems to be a still tougher and hardly tractable problem. A new problem arises; while the physicochemical functions arise with the physico-chemical structures, the biological functions must be assigned by a living organism.

Our conjecture has profound applications in the context of the genesis of cellular life. If biological functions require preconditions such as biological aims, then chemical evolution in itself cannot lead to the appearance of viable cells but to not yet alive pseudo-cells-systems having material structures identical to that of living cells, but no biological functions. Even zombie-cells, i.e., mechanically working factories of suitably assemblied systems of molecular machines, would not serve as living cells since all factories require control by intelligent living beings. Such a control is not an unimportant detail, but as, for example Noble (2008a) emphasized, it has a central importance. Moreover, the environment of living organisms is indefinitely rich in unexpected changes and so requires regularly acquired novel biological functions that are missing in mechanically working factories. We are aware of the fact that at present the prevailing paradigm is physicalism, in which even mind should be considered as "emerging" or "supervening" on the physical properties of its elementary particles (Kim 1998). Nevertheless, we point out that similarly to a sharp stone that is not a knife in the absence of teleological biological aims, a pseudo-cell is not a viable cell. The sharp and hard chopper can be produced by physical process, but its utilization as a 'knife' cannot. The point is that biological functions are teleological, and teleology cannot supervene on lower level functions determined by nonteleological physical processes. Since teleology cannot emerge from non-teleological processes, especially lawfully, and biological aims and functions are teleological, therefore a viable cell (and, similarly, mind) cannot emerge from non-teleological physical processes. Teleology is not an emergent property of physical systems (about the problem of teleology see in more details the section below-"A Deep Conceptual Problem: The Teleology of Biological Functions").

With all this we do not want to say that chemical evolution cannot be a direct precursor of biological evolution. Our conjecture only tells that chemical evolution in itself can be a necessary but not sufficient condition of cellular life.

\section{The Difference Between Physico-Chemical and Biological Symbols}

Living cells do not operate blindly (Shapiro 2011, 139). All living cells sense and respond to their environment by a set of processes known as cell signaling. They continually acquire information about the external environment and monitor their internal operations. They then use this information to guide the processes essential to survival, growth, and reproduction (ibid.). Without sign-mediated interactions no vital functions within and between organisms can be coordinated. It is exactly this feature that is absent in non-living matter (Witzany 2010). Now if functions are multilevel (Noble 2008a) and 
millions, even billions, of molecules come to function in a collective, purposeful, teleological mode that extends over distances orders of magnitude larger than individual molecules (Harold 2001, 66), the question arises how is it possible to organize millions of processes occurring at different levels into a coherent function serving biological aims.

At this important point, we find it necessary to distinguish between physical and biological symbols. Computer programs consist of physical symbols, by which we mean that once the program is running in the computer, merely physical changes occur. In contrast, we can view molecules such as cAMP as a biological symbol because there is no direct structural relationship between it and the metabolic information it represents (Shapiro 2011, 10). Cells demonstrate the capability of collecting and integrating a variety of physically different and unforeseeable signals as the basis of problem-solving decisions (Albrecht-Buehler 2009). The chemical forms are utilized as symbols that allow the cell to form a virtual representation of its functional status and its surroundings (Shapiro 2009). Cells sense their external and internal environment (Ben-Jacob, Shapira and Tauber 2006, 514), and monitor their internal states; thus cellular decisions are based on symbolic representations (Shapiro 2009, 9). If we define biological symbols by their relation to global functions of their host organism, we are consistent with Gordon Tomkins: a symbol is significant for the survival of the organism (Swan and Goldberg 2010).

In the global, cellular context in which the genetic code serves cellular functions, the activities of codemakers that are conceived as machines by Barbieri (Barbieri 2008) must be cohered with cellular activities. Therefore, even if codemaker machines could be produced somehow in the course of prebiotic evolution, they would act blindly and sporadically until the first cell develops. We argue that machines do not work until a living agent knowing how to use them acts on them. Therefore, codemaker machines work as codemakers regularly only in the context of a living organism. If in the process of postchemical (information-driven) evolution molecular machines and codemaker machines were really populating the Earth, as Barbieri (2008) claimed, then their actual working assumes the existence of a living organism capable to use, govern, and coordinate the activities of such machines and their inputs. Apparently, we are drawn into a vicious circle: codemaker machines are preconditions of cells, but their working presupposes living organisms. We obtained an important corollary: the abiogenetic scenario does not work without a biotic factor.

We argue here that the case is similar with respect to the origin of proteins and replicators, which have biologically specific information content. Chemical evolution cannot produce amino acid sequences with biological specificity in appreciable quantities, since the chemical structure must be suitable to serve biological functions. The production of such amino acid sequences would be not only extremely improbable, but also, if it did occur at all, it would be sporadic; instead of populating the earth spontaneously, decaying on a short time scale (the half-life of protein degradation for different proteins varies between a few minutes and days, Zhang et al. 2007). Therefore, if a protein with suitable biological specificity were produced in the process of prebiotic evolution, it would decay soon and so there would be no population of proteins for natural selection to act on. Similarly, a replicator must also have a suitable biological specificity, and so it could be produced only rarely. Moreover, macromolecules like the DNA in cells after death within conditions suitable for cellular life degrade on a timescale less than a few days or weeks (Mona et al. 2008, Nicklas, Noreault-Conti and Buel 2012 , Itani et al. 2011). Again, we find that the chemical evolution scenario of cellular biogenesis suffers from basic insufficiencies. Moreover, a replicator is merely an instrument of replication; and until a living organism uses it regularly for replication, the replicator does not replicate itself as a rule. Similar is the case in postchemical evolution in which molecular machines are already present and can produce molecules with highly specific information content, for the molecular machines in the absence of cells are not capable of self-initiating the activities necessary to produce such replicators regularly. And even if they were, such replicators would not have biological functions arising from molecular machines, since in a physical process molecular machines cannot produce and assign teleological biological functions.

Barbieri (2012) argues that although the 'chemical paradigm' is very popular today and is often considered to be in agreement with the Darwinian paradigm, this is not the case. The reason is that natural selection, the cornerstone of Darwinian evolution, does not exist in inanimate matter (ibid.). From the $1950 \mathrm{~s}$ and $60 \mathrm{~s}$, it became clear that biological information and the genetic code were fundamental and unique to living systems. Now the question is not only how the chemical constituents of the cell arose, but also, how did biologically specific information and code appear? Moreover, besides the problem of how vital information arises, we have to face also the problem of how utilization of information for biological purposes arises. Whether the information present in cellular symbols is actually 'read' and executed by cellular effectors depends on membrane-bound activity. All 
major activities of cells are topologically connected to membranes (Hoffmeyer 1999), and so, to the global level of the cell.

As Harold (2001) noted, from the chemistry of macromolecules and the reactions they catalyze, little can be inferred regarding their articulation into physiological functions at the cellular level (ibid, 5). This highly indirect connection between the chemical properties of macromolecules and their biological roles in serving physiological functions presents a fundamental problem for chemical evolution scenarios. Indeed, no one has yet discovered a persuasive chemical connection between any particular triplet of nucleotides and the amino acid that this triplet codes for (ibid., 58). Similarly, Barbieri (2008) argued that there is no (physically - addition by the present author) deterministic link between codons and amino acids. Therefore, it seems that the genetic code representing a rule connecting particular triplets of DNA or RNA nucleotides and amino acids of proteins cannot arise from physico-chemical laws. We call attention to the fact that a biological rule cannot arise from physico-chemical processes, especially from random processes. A rule cannot emerge from phenomena but from law. Therefore if the rule present in the genetic code does not arise from physico-chemical processes and laws, the only alternative is that it arises on the basis of biological laws or principles the more so because the genetic code must serve, utlimately, biological functions.

This means that it is not chemical law but biological law or principle that governs the development of biologically specific sequences, biological rules and biological codes. This result indicates that the theory of terrestrial abiogenesis relies implicitly on the presence of a biotic factor before the appearance of the first cells on Earth. In a strict sense, abiogenesis is not completely abiotic, even if its biotic part has remained until now unnoticed. This is our second argument against the purely physicochemical origin of cellular life.

\section{Symbols, Rules and Codes}

The Oxford English Dictionary tells that a symbol is something used for or regarded as representing something else; a material object representing something, often something immaterial. Therefore, we can consider that biological symbols are physico-chemical objects representing biologically specific, useful information (meaning) in the context of the hierarchical organization of teleological biological aims (functions). "The amazing property of symbols is their ability to control the lawful behavior of matter, while the laws, on the other hand, do not exert control over the symbols or their coded references" (Pattee and Kull 2009). As we defined above, biological symbols are not determined by their local physico-chemical structures even if these must be suitable to serve as tools and realize biological aims. It is biological meaning that makes biomolecules suitable to serve global, cellular biological functions, realizing biological governance. Biological meanings must be real things having a causal power on physical matter. Such meanings must exist in a way suitable to be coupled to any other relevant biological meanings, be guided on the basis of their meanings, and therefore coupled in a physically arbitrary manner. Such symbols having biological governance can harness physical laws at the same time that the physico-chemical biomolecules obey physico-chemical laws in their immediate, local and instantaneous physical conditions. Nevertheless, from time-step to time-step they must deviate in a progressively increasing rate from physical behavior, in a rate allowed by quantum undeterminacy, in order to serve biological functions.

We emphasize that there is a fundamental physical fact allowing biological determinations. We propose that the microscopic door of quantum indeterminacies allows biological determinations within the limits of the Heisenberg uncertainty relation written for virtual processes in the form $\Delta \mathrm{E} \Delta \mathrm{t} \leqq \mathrm{h} / 2 \pi$ (where $\Delta \mathrm{E}$ is the energy of the instantaneously created virtual quanta and $\Delta \mathrm{t}$ is the time interval of their existence, see Grandpierre 2012, Grandpierre and Kafatos 2012). The "region of indeterminacy" in which life can establish itself (Pattee and Kull 2009) is, by our proposal, given by virtual processes allowed to be generated by the narrow range of the uncertainty relation. The hypothesis about the causal closure of the physical world, by our proposal, should be replaced by the more general thesis of the causal closure of the natural world, where the term 'natural' involves not only the physical but biological (and, possibly, psychological) causes as well. Biological causes can actually control physical processes because they can act on the chain of physical causes through the quantum door. With the help of such virtual processes microscopic biological interventions can add up as a collective phenomenon so that biological behavior can systematically deviate from the physical one that would occur within the same conditions in the absence of biological deterinations (Grandpierre 2012, Grandpierre and Kafatos 2012).

Our proposal sheds new light on the results of Boi (2010) telling that the spatial and geometrical or topological changes of biomolecules are not predetermined. Boi (2010) adds that the structure of 
biomolecules depends significantly on dynamic phenomena that organize and integrate it. CruzeiroHansson (2001) noted that proteins are devices that are able to transduce initial quantum excitations to the generation of concerted motions of a large group of atoms. Biological symbols act in the context of the astronomically large number of particles and energetic quanta that represent a collective quantum state. In this way, biological aims with the help of biological symbols and the time-variable signaling network can act on physical processes and harness them (Polanyi 1968).

As a second step, let us turn to rules. As the Oxford English Dictionary tells, rules are laws or principles that operate within a particular sphere, they are principles governing conduct within a particular activity or sphere, describing or prescribing what is possible or allowable. We consider that biological rules are biological laws or principles that operate in the particular sphere of biological meanings of biological symbols. Barbieri claimed that "there is no deterministic link between codons and amino acids, and a one-to-one correspondence between them could only be the result of conventional rules". Taking into consideration that conventional rules are based on conventions, and prebiotic objects cannot make conventions, we find there is a need to ground the link between codons and amino acids in biological laws or principles (in more detail, see the section About the Biological Principle).

As a third step, let us consider codes. As the Oxford English Dictionary writes, a code is a system of words, letters, figures, or other symbols substituted for other words, letters, etc. We consider that codes are rules connecting two sets of symbols. Substituting here the above definition of 'rule', we obtain that biological codes are biological laws or principles determining the connections between symbols, by which the genuinely biological activities of the cell are governed. This definition fits to the definition of the Free Dictionary (entry 'code', 2012): A code is a systematically arranged and comprehensive collection of laws.

It is useful to add another important distinction. While codes are conceived as being just prescriptions between possible or allowed actions, they are still merely passive tools. Physical codes like computer codes cannot initiate their activities. In contrast, in a real living cell codes are biologically active. A biological code is active by the biological law or principle. We note that since the biological principle (Bauer 1967/1935, 51) corresponds only to one basic and global physical parameter, namely, to the thermodynamic distance of the organism as a whole from equilibrium, all the other remaining physical degrees of freedom must be decided on the basis of biological autonomy (Grandpierre and Kafatos 2012) by the living organism itself. This means that the biological principle and biological autonomy are inseparable. Freedom at the molecular and cellular levels allows autonomy and spontaneity to emerge even in single celled organisms (Kawade 2009).

Therefore, at the end of the causal chain, besides (i) symbols, (ii) rules and (iii) codes, we find as a fourth, actually, twofold ingredient (iv) the biological principle and biological autonomy as the agents using biological tools. This means that for a cell to be viable, biological principle and biological autonomy must be present. We point out that the biological principle and autonomy together are also suitable to serve as the basis of the third factor, the biological code. Moreover, they can yield the second factor as well, the biological rules that operate within living organisms. Instead of assuming a strange 'convention' to establish these rules, our proposal is based on a natural principle. Additionally, this fourth ingredient can be responsible for producing biological symbols, assigning biological functions to cellular ingredients, yielding the first factor of biological organization. This fourth ingredient can be identified as being itself the ultimate biological aim, flourishing. Therefore, the introduction of biological principle and genuine biological autonomy into the vocabulary of biologists seems not only explanatory but also economic. We obtained a third argument against a purely physicochemical origin of cellular life on the basis of our analysis of symbols, roles and codes.

We emphasize that biosemiotic governance of the cell's behavior is not the result of additional constraints on physical processes, as it is widely believed (see e.g., Pattee 1969, 161, Noble 2008b), but serve biological aims and arise through the door of quantum indeterminacy, which opens new physical possibilities that do not arise in an otherwise identical but inanimate physical system.

We note that such a proposal offers a way to escape the circularity of the popular scenarios in which cellular life is explained by the information paradigm, while the origin of biological information presupposes the presence of life. Our solution works without such circularity because such a deep biological principle of nature should not originate in the process of cellular biogenesis, since fundamental laws of nature exist independently of the origin of cellular life. Similarly to physics, where gauge fields manifesting fundamental physical laws are the messengers carrying signals between elementary particles that enable them to interact with each other (Huang 2007, xi), in biology also a fundamental law of nature - a biological principle — is what guides the signals that coordinate cellular activities. The circularity can be escaped by allowing more general cosmic life forms existing 
before cellular life. Therefore, by our results, one of the central statements of cell theory, "all cells are from cells", should be generalized to "all life is from life".

\section{A Deep Conceptual Problem: The Teleology of Biological Functions}

We define biological functions as coherent systems of biological processes serving biological aims, ultimately, flourishing (the tendency towards the fittest or highest vitality) of the organism as a whole. A cell cannot be alive if its proteins do not have their global, biological functions, like cell signaling orchestrating cellular activities (Berridge 2012). As the entry "biology" in the Encyclopaedia Britannica (Green 2012) states, living organisms cannot exist without biological functions: "Living things are defined in terms of the activities or functions that are missing in nonliving things." While functions are basic in biology, they are problematic from a physical point of view. The problem arises because biological functions are teleological, and teleology seems to be inconsistent with the conceptual scheme of physics: "The biological concept of function appears teleological, implying goal directedness or purpose.... Ever since the scientific revolution, however, teleology has become exiled from science" (Buller 2002, 393). But why does it seem that teleology is inconsistent with physics? We argue that the reason is that biological teleology cannot arise in a Laplacian deterministic universe, since in such a case the only type of determination permitted is the one prescribed by deterministic differential equations of physics applied to given physical conditions. We point out that beyond the level of differential equations, which describe only the local picture, physics has worked out a yet deeper grasp of problems with the help of integral principles (the least action principle). The least action principle posits a global picture grasping the whole physical path between a given initial and an endpoint. Moreover, since the invention of quantum physics we know that our universe is not completely determined by physical conditions and laws. We proposed above that quantum indeterminacy opens up a way that permits logical consideration of a new type of determination, namely, biological determination that serves biological aims. Since we know that biological functions exist, biological teleology must also exist; and the only way to justify biological teleology is to allow the biological determination of what quantum physics left undetermined. Biological teleology is such a basic fact of nature that, as it is formulated recently, "Nothing in biology makes sense, except in the light of teleology'. This could be the first sentence in a textbook about the methodology of biology" (Toepfer 2012).

Still, how do we ground biological teleology within the natural sciences? Our proposal is to generalize the already somewhat teleological least action principle (which itself involves only an apparent teleology, since the endpoint of the least action principle is pre-fixed by the physical conditions); and to allow the endpoint to be variable, so that the endpoint of the generalized action principle can be regarded as biologically determined (Grandpierre 2007). Since biological interventions are collective phenomena, at the quantum level they can add up to macroscopic amplitudes, and so biological endpoints can differ significantly from physical ones (Grandpierre 2012). In this way, we found a physical basis to allow biological teleology.

\section{Biological Principle and Teleology}

"The temporality of living things is guided by the purpose to live, which works as the semantic boundary condition for the processes of the embodiment of subjectivity" (Kawade 2009). As Noble (2008a) pointed out, there are no sequences of instructions in the genome that could possibly play a role similar to that of a computer program. The reason is very simple: A database, used by the system as a whole, is not a program. The networks of events that might be interpreted as programs are themselves the functions we are seeking to understand. There is no separate program (Noble 2008a). Then what drives the time-variable network of events that represent an important form of biological information? Acknowledging that time-variable behavior must be governed by the laws of motion within the given input conditions, the identity of dynamic global biological information and the timevariable behavior of organized events seem to imply that biological behavior is informed by the biological principle (see the section below: About the Biological Principle). In every time-step, within quantum limits, the input conditions to the physical laws are modified by the biological principle to govern physical processes towards the endpoints of the biological action principle.

Independent arguments also show that chemistry cannot determine biological functions. As Davies (1999, 237) argued: genomes are more or less random sequences of base pairs, and this very randomness is essential if they are to play the role of evolvable, information-rich molecules. But this 
fact flatly contradicts the claim that genes can be generated by a simple, predictable, law-like physical process. A law of nature of the sort that we know (physical law) will not create biological information, or indeed any information at all. Contrary to the oft-repeated claim, then, life cannot be 'written into' the laws of physics - at least, not into anything like the laws of physics that we know at present. The whole point of the genetic code, for example, is to free life from the shackles of non-random chemical bonding. Life works its magic not by bowing to the directionality of chemistry, but by circumventing what is chemically and thermodynamically expected (ibid.). Of course, organisms must comply with the laws of physics and chemistry; but these laws are only incidental to biology (Davies 1999, 236237). All these (and many other) facts underpin the finding that biological determinations can be free, within certain limits, from physical constraints.

\section{On the Evolution of Biological Aims}

Since biological aims are harnessing physical processes, they must come first. Instead of the genesis of material structures, one must study the genesis of biological aims first. Abiogenesis assumes that the ultimate biological aim (like survival) arises from simple ones like bond-making and catalysis. Yet we point out that such simple, physical functions are not only subservient to survival, but also highly inert. The popular hypothesis of the origin of biological functions, also shared by Kawamura (2012), assumes that the biological functions of RNA are automatically given by the formation of their spatial structures. We argue that such a hypothesis should be revised. By our argument, cellular life cannot evolve from chemical or post-chemical (i.e., driven by physical information, like in computers) evolution. The main reason is that biological functions involve highly sophisticated multilevel orchestration of biochemical and biological processes that are teleological. Therefore, they cannot arise from local, physico-chemical functions. Just as the hammersmith does not develop from hammers, and hammers cannot populate the earth before the appearance of hammersmiths, biological functions cannot develop from the assumed evolution of subservient, physical functions. We think that, in the era of systems biology, it is timely to realize that all biological functions arise from the fundamental biological aim to flourish, or in other words, to prosper. If so, this fundamental biological aim must come first in the cosmic context of cellular biogenesis, and cellular life must develop directly from it. We obtained a fourth argument against a purely physico-chemical origin of cellular life: the argument from the evolution of biological functions.

Considering the origin of cellular life in this context leads us to the problem of the origin of this fundamental biological aim to flourish. We propose grounding this apparently hard to grasp biological aim in a scientific context by identifying or associating this fundamental biological aim with the biological principle. Since the fundamental laws of nature like the biological principle need not necessarily arise in time, the biological principle does not have to originate from previous circumstances. In this way, we are led to a novel context in the search for the origin of life, corresponding to a time-scale transcending that of most present-day physical cosmologies assuming the origin of the universe in the Big Bang. The apparent conflict of eternal biological principle and finite Big Bang universe may be resolved if one recalls that the Big Bang may arise from quantum vacuum fluctuations and so the quantum vacuum may precede the Big Bang. Therefore, the eternal character of the biological principle may correspond to the similar character of the quantum vacuum. Indeed, on the basis of Bauer's principle it has been shown that the quantum vacuum could be conceived as a kind of cosmic life form (Grandpierre 2008).

\section{About the Biological Principle}

We thus arrive at the deepest and most troublesome conceptual depth of the natural sciences, one that requires special attention, the question that humanity regards as the 'Mother of all questions': the essence and origin of life (Pályi et al. 2002). Ervin Bauer, the founder of the first, exactly formulated theoretical biology, who is still practically unknown for mainly historical reasons, presented a universal law of all living organisms also in mathematical form. This law requires the permanent selfinitiation of work investment against the equilibration that would otherwise occur within the given initial and boundary conditions on the basis of the physico-chemical laws (Bauer 1967, 51). From this universal law of biology, frequently called Bauer's principle, Bauer was able to derive all fundamental life phenomena - such as metabolism, growth, regeneration, and death - in a quantitative manner. His principle holds that the basic aim of living organisms is not only survival per se, but also that the organism maintains distance from the lethal thermodynamic equilibrium in as high a degree as possible 
and for a period as long as possible. This means that in the idea of survival, only one aspect of the primary biological aim is grasped, since the other aspect is to live life with preferably high vitality. We associate these simultaneously present two aspects, survival and high vitality, together with the term 'flourish'. We mention that since the quantity "action" in the least action principle has a dimension of energy times time, the tendency of living organisms towards living as long as possible and as high above equilibrium as possible can be expressed in a mathematical form as the greatest action principle (Grandpierre 2007). We note that this primary, fundamental biological aim, flourishing, corresponding to a given living organism, cannot stand alone. In order to become realized, it must produce a whole system of subservient biological aims so that all the inevitable tasks necessary to maintain life and flourish should be realized, from the molecular to the organismal level. Considering that between the molecular and organismal levels intermediate levels of biological organization occur, it can be easily shown that all biological aims of a living organism form a hierarchical system, all rooted in the fundamental aim to flourish.

We are speaking of genuine biological teleology — of something that is missing in contemporary physics. It becomes increasingly apparent that life and consciousness cannot originate from physics. More concretely, biological functions like 'flourishing' are teleological, and so they are something additional to physical properties. Moreover, since Bauer's principle can be formulated as the greatest action principle (Grandpierre 2007), in a way that is the generalized form of the least action principle allowing free endpoint selection, it is apparent that the biological principle cannot be derived from its special case, which is the physical principle.

If the biological principle acts everywhere in the universe, similaly to the physical principle, then within any conditions the biological principle acts towards conditions favorable for life. Therefore, evolution of the universe is guided towards higher and higher openness by the biological principle, in a way increasingly different from Laplacian determinism, but not so much towards a different physical trajectory of the physical objects' center of mass, but towards increasing complexity, vitality and flourishing. If biological laws act everywhere, this would offer an explanation to certain otherwise hardly explainable experimental facts. For example, the experiments of Steinman and Cole (1967) seemed to confirm that molecules significant for life are made preferentially. They noted that "preferential interaction has been observed at higher levels of organization as well," going so far as to allege that "a sort of built-in 'predestination' can be identified at general levels of biological order." Moreover, the late Cyril Ponnamperuma, who, like Sidney Fox, was one of the early pioneers in biogenesis research, believed that "there are inherent properties in the atoms and molecules which seem to direct the synthesis towards life" (Shapiro 1986, 186-187).

\section{On the Origin of Mind}

Life's basic command is: flourish! Such a command has a remarkable property that apparently escaped due attention: it represents an extraordinary conceptual depth. The command "flourish!" is not a concrete, physical, but a conceptual, general command, that, in order to become realized, first it should be "solved" how to apply it to the concrete situation, what kind of concrete physical processes are consistent with it and how to select from all the quasi-equivalent sets of these possible processes within the given spatial and temporal conditions. Such an activity requires an overview of the external and internal environment, their virtual, symbolic representation and their correspondance to the basic biological aim, and decision at that symbolic level. The realization of the basic, conceptually deep biological aim "flourish!" requires a well-organized hierarchical set of subservient biological aims that can be realized - as we argued above - only with symbols. Assigning these subservient set of biological aims requires continuous creation of signs. Organizing biological functions into a viable unit requires biological symbols. It is signaling through which orchestration of cellular activities into a functioning unit realizes the basic biological aim. Organization of all symbolic activities into a viable unit can be regarded as a formulation of mind. We arrive to the result that assigning aims, cohering them into a conceptually deep-levelled biological aim and deciding about behavior accordingly is the basic and distinguishing property of mind. This means that mind is an inevitable tool of life. Therefore, any progress in the solution of the problem regarding the origin of life is also a progress regarding the nature and origin of mind.

\section{Acknowledgment}


It is a pleasure for me to express my thanks to my friend Jean Drew for the galvanizing discussions and correcting the English of this paper.

\section{References}

Albrecht-Buehler, G. (2009). 'Cell Intelligence'. Available at http://www.basic.northwestern.edu/g-buehler/FRAME.HTM [15 July 2012].

Barbieri, M. (2008). Life is Biosemiosis. Cosmos and History: The Journal of Natural and Social Philosophy, 4 (1-2): $29-.52$.

Barbieri, M. (2012). The Paradigms of Biology. Biosemiotics, April 2012, 1-27.

Bauer, E. (1967). Theoretical Biology. Akadémiai Kiadó: Budapest, Hungary (in Hungarian. 1967; in Russian, 1935, 1982, 1993, 2002).

Beckner, M. (1969). Function and Teleology. J Hist Biol 2: 151-164.

Ben-Jacob E., Shapira Y., Tauber A. I. (2006). Seeking the Foundations of Cognition in Bacteria: From Schrödinger's Negative Entropy to Latent Information. Phys A 359: 495-524.

Berridge, M.J. (2012). Cell Signalling Biology; doi:10.1042/csb0001001

Boi, L. (2010). Méthodes mathématiques, processus biologiques et philosophie de la nature. Eikasia. Revista de Filosofía, VI (35): 267-297.

Bouchard, T. J. (2004). Genetic influence on human psychological traits: A survey. Current Directions in Psychological Science, 13: $148-151$.

Buller, D. J. (2002). Function and Teleology, in: Encycl. Life Sci. (London: Macmillan), p. 393.

'code', 2012, entry, Oxford English Dictionary

Cruzeiro-Hansson, L. (2001). How Do Proteins Work? Proc. First European Workshop on Exo/Astro-Biology. Frascati 21-23 May 2001. ESA SP-496.

Dar-Nimrod I, Heine S. J. (2011). Genetic essentialism: on the deceptive determinism of DNA. Psychol Bull. 137(5): 800-18.

Davies, P. (1999). The Fifth Miracle. The Search for the Origin of Life. Penguin: London.

Grandpierre A. (2007). Biological Extension of the Action Principle: Endpoint Determination beyond the Quantum Level and the Ultimate Physical Roots of Consciousness. Neuroquantology, 5: 346-362.

Grandpierre A. (2008). Cosmic Life Forms. In: From Fossils to Astrobiology. Seckbach J and Walsh M (Eds), (Berlin: Springer), pp. 369-385.

Grandpierre, A. (2012). "Genuine Biological Autonomy: How can the Spooky Finger of Mind play on the Physical Keyboard of the Brain?" Athens: ATINER'S Conference Paper Series, No: PHI2012-0197. http://www.atiner.gr/papers/PHI2012-0197.pdf Grandpierre, A. and Kafatos, M. (2012). Biological Autonomy. Philosophy Study, 2(9): 631-649.

Green, Edna R. (2012). entry: „biology.” Encyclopædia Britannica. Encyclopædia Britannica Ultimate Reference Suite. Chicago: Encyclopædia Britannica.

Harold, F. M. (2001). The Way of the Cell. Molecules, Organisms and the Order of Life. Oxford University Press: Oxford.

Hoffmeyer, J. (1998). Surfaces inside surfaces: On the origin of agency and life. Cybernetics and Human Knowing 5 (1), $33-42$.

Hoffmeyer, J. (1999). Order out of indeterminacy. Semiotica 127-1/4 (1999), 321-343.

Hoffmeyer, J. (2001). Life and reference. BioSystems 60: 123-130.

Huang, K. (2007). Fundamental Forces of Nature. The Story of Gauge Fields. World Scientific: Singapore.

Itani, M. Yamamoto, Y. Doi, Y. Miyaishi, S. (2011). Quantitative Analysis of DNA Degradation in the Dead Body. Acta Medica Okayama 65: 299-306.

Kawade, Y. (1992). A molecular semiotic view of biology. Interferon and 'homeokine' as symbols. Rivista di Biologia - Biology Forum 85(1): $71-78$.

Kawade, Y. (2009). On the Nature of the Subjectivity of Living Things. Biosemiotics 2.2: 205-220.

Kawamura, K. (2012). Drawbacks of the ancient RNA-based life-like system under primitive earth conditions. Biochimie 94 (7): $1441-1450$.

Kim, J. (1998). Mind in a Physical World. Cambridge, Massachusetts: The MIT Press.

Mona, A. E-H., Sahar A. E-D., Sohayla, M. A., Nermin, A. H., Sobhy, E. H. E-N. (2008). The Relationship between Postmortrem Interval and DNA Degradation in Different Tissues of Drowned Rats. Mansoura J. Forensic Med. Clin. Toxicol. XVI: 45-61.

Nicklas, J. A., Noreault-Conti, T. and Buel, E. (2012). Development of a Real-Time Method to Detect

DNA Degradation in Forensic Samples. J Forensic Sci, 57(2): 466-471.

Noble, D. (2008a). Claude Bernard, the first systems biologist, and the future of physiology. Exp Physiol 93.1: 16-26.

Noble, D. (2008b). Genes and causation. Phil. Trans. R. Soc. A 366: 3001-3015.

Noble, D. (2010). Biophysics and systems biology. Review. Phil. Trans. R. Soc. A 368: 1125-1139.

Pályi, G., Zucchi, C. and Caglioti, L. (Eds.) (2002). Fundamentals of Life. Paris: Elsevier.

Pattee, H. H. (1973). The Physical Basis and Origin of Hierarchical Control. In: Hierarchy Theory: The Challenge of Complex Systems. H. H. Pattee (ed.). New York: Braziller (pp. 73-108).

Pattee, H. H. and Kull, K. (2009). A biosemiotic conversation: Between physics and semiotics. Sign Systems Studies 37(1-2): 311-331.

Polanyi, M. (1968). Life's Irreducible Structure. Science 160: 1308-1312.

Rosoff, P. M. (2012). The myth of genetic enhancement. Theor Med Bioeth (2012) 33: 163-178.

Shapiro, R. (1986). Origins: A Sceptic's Guide to the Creation of Life on Earth. Summit Books, New York,186-7.

Shapiro, J. A. (2009). Revisiting the Central Dogma in the 21st Century. Natural Genetic Engineering and Natural Genome Editing: Ann NY Acad Sci 1178: 6-28.

Shapiro, J. A. (2011). Evolution: : A View from the 21st Century. FTPress Science.

Steinman, G. And Cole, M. (1976). Synthesis of biologically pertinent peptides under possible primordial conditions. Proceedings of the National Academy of Science 58, 735.

Swan, L. S. and Goldberg, L. J. (2010). Biosymbols: Symbols in Life and Mind. Biosemiotics 3:17-31.

Tachibana, C., White, A. and Johnson, N. A. (2010). Rediscovering Biology. Proteins and Proteomics. http://www.learner.org/courses/biology/textbook/proteo/proteo_4.html 
Toepfer, G. (2012). Teleology and its constitutive role for biology as the science of organized systems in nature. Studies in History and Philosophy of Science Part C: Studies in History and Philosophy of Biological and Biomedical Sciences 43 (1): 113 119.

Witzany, G. (2010). Biocommunication and Natural Genome Editing. Springer: Berlin.

Zhang, L. Gurskaya, N. G., Merzlyak, E. M., Staroverov, D. B., Mudrik, N. N., Samarkina, O. N., Vinokurov, L. M., Lukyanov, S. and Lukyanov, K A. (2007). Method for real-time monitoring of protein degradation at the single cell level. BioTechniques 42(4): 446-450. 